\title{
Stability of traditionally processed vegetable oils and their blends under different storage conditions
}

\author{
Faustine N. NGASSAPA ${ }^{1}$, Tupeligwe R. MWAISAKA ${ }^{2}$ and Stephen S. NYANDORO ${ }^{*}$ \\ ${ }^{I}$ Chemistry Department, College of Natural and Applied Sciences, University of Dar es Salaam, P.O. box \\ 35061, Dar es Salaam, Tanzania. \\ ${ }^{2}$ Government Chemist Laboratory. Lake Zone Labotatory, P.O.Box 502 Mwanza, Tanzania. \\ ${ }^{*}$ Corresponding author; E-mail: nyandoro@udsm.ac.tz; samnyandoro@yahoo.com;
}

Tel : +255-754-206560, +255-718944724

\begin{abstract}
The objective of the study was to investigate the stability of traditionally processed palm oil (PO), sunflower oil (SO) and sesame oil (SSO) and their blends as function of storage conditions by analysing their physicochemical properties which included acid value, saponification value, peroxide value, iodine value and refractive index. The oils and their blends were stored in two different conditions; one batch at the air-tight dark conditions, while another batch at the air free-ambient, both for 60 days. Sampling was done once after 10 days and the analyses conducted using standard methods. Samples stored at the air-tight dark conditions remained relatively stable than those at the air free-ambient temperature, with SSO showing the highest stability under the studied storage conditions. Generally, the blends showed better stability than the unblended, with some few exceptions, formulations having higher proportions of SSO showed desirable qualities based on physicochemical parameters evaluated. Hence, such traditionally processed vegetable oils should be specially packed to keep them free from the light and air for prolonged storage. The study further reveals the significance of blending in improving the quality and stability of vegetable oils.
\end{abstract}

(C) 2016 International Formulae Group. All rights reserved.

Keywords: Palm, Sunflower, Sesame oil, Blends, Traditionally processed oils, Storage, Stability.

\section{INTRODUCTION}

Most native vegetable oils and fats have limited applications in their unmodified forms, imposed by their triacylglycerol (TAG) and fatty acid (FA) compositions. Changing the natural physicochemical characteristics of oils or fats provide greater functionalities of a large number of product formulations. Thus, oils and fats applications can be widened by modifying them through blending, fractionation, inter/trans-esterification, hydrogenation or combinations of these processes (Myat et al., 2009; Abdulkarim et al., 2010). Blending is the simplest and the most feasible method used to modify oils and fats for enhancing their oxidative stability and other functionalities for optimizing their application in food products without necessity of chemical treatment (Myat et al., 2009; Naghshineh et al., 2009; Siddique et al., 2010).

A number of traditionally processed vegetable oils such as palm, sesame and sunflower oils are marketed in Tanzania and 
used in various food preparations (Sulle and Nelson 2009; Ngassapa et al., 2012). Notwithstanding their popular use in food preparations, the stability and associated physicochemical changes taking place during the storage of these traditionally processed vegetable oils and their blended forms have not been thoroughly assessed. With the rising concerns over the natural products and emphasis on nutritional enrichment, blending of vegetable oils and fats has emerged as an economical way to produce edible oils devoid of any chemical treatment, hence retaining natural flavour, nutritional value and other desirable characteristics (Myat et al., 2009). The nutritional properties of importance are based on essential fatty acid contents, omega3 to omega- 6 ratio, saturated to unsaturated ratio and contents of antioxidants, monounsaturated and polyunsaturated (PUFAs) fatty acids (Finley and Shahidi, 2001; WHO, 2003; Lee and Hwang, 2008; Myatet al., 2009).Such nutritional and general oil quality parameters can be enriched by blending among other approaches (Serjouie et al., 2010; Naghshineh et al., 2009; Siddique et al., 2010; Ngassapa et al., 2012). It was therefore anticipated that oxidative stability of the traditionally processed vegetable oils could be improved by blending them to obtain good and desirable formulations which will help to improve their storage stability. Thus, the present study was undertaken to investigate the stability of traditionally processed palm, sunflower and sesame oils and their blends as function of storage conditions by analysing their physicochemical properties which included: acid value, saponification value, peroxide value, iodine value and refractive index.

\section{MATERIALS AND METHODS Oil samples collection}

Three types of traditionally processed palm (Elaeis guineensis), sesame (Sesamum indicum) and sunflower (Helianthus annuus) oils were purchased from the local producers in Kyela District, Mbeya Region (palm oil), Kondoa District, Dodoma Region (sunflower oil) and Masasi District, Mtwara Region (sesame oil) in Tanzania. The sampling and storage procedure were conducted as described by TZS 51 (Part I): 1979 and TZS 561: 2001. The samples were transported to the Analytical Laboratory of the Chemistry Department, University of Dar es Salaam for storage and analyses.

\section{Preparation of oil blends, storage and treatment conditions}

Oil blends were prepared by mixing palm oil with sunflower or/and sesame oils at a ratio of $3: 1,1: 1$ and $1: 3(\mathrm{~W} / \mathrm{W})$ for binary blends and 1:1:1, 2:1:1, 1:2:1 and 1:1:2 (W/W) for ternary blends. The mixture was magnetically stirred for 20 minutes to allow homogenization. The first batch of oils and their blends was sealed and stored in the air tight-dark conditions, while the second batch was stored at air free-ambient temperature for 60 days. Sampling for analysis was performed once after 10 days for the 60 days of storage time. Analytical grade reagents were used. All glassware were cleaned with liquid soap, rinsed with distilled water and then oven-dried before use.

\section{Analyses of physicochemical properties}

Five physicochemical properties named Acid value (AV), saponification value (SV), peroxide value (PV), iodine value (IV) and refractive index (RI) were determined.

\section{Acid value}

TZS 51 Part 1: 1979 and TZS 561: 2001 protocols were adopted for determination of acid value. A drop of phenolphthalein (POP) indicator (1\% solution in alcohol) was added to $50 \mathrm{ml}$ ethanol-ether mixture $(1: 1 \mathrm{v} / \mathrm{v})$. To the mixture, $10 \mathrm{~g}$ of unblended sesame and sunflower oils were added then boiled on hot plate for 5 minutes. The mixed solvent was titrated against $0.1 \mathrm{~N}$ $\mathrm{KOH}$ solution until a pink colour that persisted for about 30 seconds was formed. For palm oil and its blends, $20 \mathrm{~g}$ of the sample weighed into a flask followed by addition of $50 \mathrm{ml}$ of neutralized isopropanol. The 
resulting mixture was placed on the hot plate at $40{ }^{\circ} \mathrm{C}$ for 5 minutes. The sample was titrated against $0.1 \mathrm{~N} \mathrm{KOH}$ to the first permanent pink colour.

\section{Saponification value}

The TZS 561:2001 procedure was adopted whereby $2 \mathrm{~g}$ of the sample was weighed in a conical flask followed by addition of $25 \mathrm{~mL}$ of alcoholic potassium hydroxide. The flask was connected to the reflux air condenser and heated on electric heating mantle for about 1 hour. The sample was heated gently but steadily until the sample was completely saponified as indicated by the appearance of clear solution. After the flask and condenser have cooled, they were washed with little water followed by addition of POP indicator and then titrated against $0.5 \mathrm{~N}$ hydrochloric acid. The blank test was carried out in parallel with the SV determination.

\section{Peroxide value}

TZS 561: 2001 and TZS 51 Part 1: 1979 protocols were adopted in which $5 \mathrm{~g}$ of the sample was weighed into the $250 \mathrm{ml}$ flask followed by addition of $30 \mathrm{ml}$ of the acetic acid-chloroform $(3: 2 \mathrm{~V} / \mathrm{V})$ solution and the flask swirled until the sample was dissolved in the solution. $0.5 \mathrm{~mL}$ of saturated potassium iodide was added and swirled for 1 minute followed by addition of $30 \mathrm{~mL}$ of distilled water. Titration with $0.01 \mathrm{~N}$ sodium thiosulphate solution was carried out with constant and vigorous shaking until the yellow colour has almost disappeared. Thereafter, about $0.5 \mathrm{ml}$ of starch indicator solution was added and titration continued until the blue colour has just disappeared. The blank test was carried out in the parallel with the PV determination.

\section{Iodine value}

Wijs' method procedure given by TZS 51 Part 1:1979 and TZS 561:2001 was adopted whereby $0.4 \mathrm{~g}$ of the sample was weighed into a clean dry $500 \mathrm{~mL}$ iodine flask to which $25 \mathrm{~mL}$ of chloroform have been added and agitate to dissolve the contents. 25
$\mathrm{mL}$ of Wijs solution was added and the glass stopper was replaced and swirled for intimate mixing and allowed to stand in the dark for 30 minutes (for sesame and sunflower oil) and 1 hour for palm oil and the blends. After standing for above stated time, $15 \mathrm{~mL}$ of potassium iodide solution and $100 \mathrm{ml}$ of water were added in sesame and sunflower oil samples while in palm oil and blends $20 \mathrm{ml}$ of potassium iodide and $150 \mathrm{ml}$ of water were added. The liberated iodine was titrated with $0.1 \mathrm{~N}$ sodium thiosulphate until the colour of the solution was straw yellow followed by addition of one millilitre of the starch solution and the titration continued until the blue colour formed disappeared after thorough shaking with the stopper on. The experiment was carried in duplicates and the blank test was carried out simultaneously under similar experimental conditions.

\section{Refractive index}

The refractive index of oils and fats was measured by refractometer at a constant pressure. The TZS 51 Part 1:1979 and TZS 561:2001 protocols were followed. Abbe refractometer connected to the thermostatically controlled at $40{ }^{\circ} \mathrm{C}$ water bath with a circulating pump was used. Two drops of sample were placed on the lower prism. The prism was closed, tightened firmly with the screw-head, and allowed to stand for 1-2 minutes after which the determination of RI was done. Ethanol was used for cleaning the prism of the refractometer.

\section{Data analysis}

Duplicate sample sets of pure oils and blends were analysed and the mean values calculated for each physicochemical parameter determined. Mean and Standard deviations of $\mathrm{AV}, \mathrm{SV}, \mathrm{PV}, \mathrm{IV}$ and RI were calculated using excel computer package. Instat3 statistical package was used for multiple comparison with one way ANOVA/ttest to establish the significant difference within one type of oil, across various oil types and their blends as functions of storage conditions $(\alpha=0.05)$. 


\section{RESULTS}

Change in acid value (AV), saponification value (SV), peroxide value $(\mathrm{PV})$, iodine value (IV) and refractive index (RI) of the studied vegetable oils and their blends after 60 days of storage under the air tight-dark conditions and at the air freeambient temperature are presented in Table 1, 2, 3, 4 and 5, respectively. AV for unblended $\mathrm{PO}, \mathrm{SO}$ and SSO showed the significant difference $(p<0.05)$ in an increase of FFA content after 60 days of storage time in all storage conditions whereby SSO appeared to be the most hydrolytically stable oils. The storage conditions had also effect on the FFA content of all blends as shown in Table 1.The storage conditions had significant effect $(p<0.05)$ on the SV for both unblended PO, SO and SSO and blended oils(Table 2). The $\mathrm{PV}$ of unblended PO, SO and SSO showed an increase in both storage conditions, PO being marginally least affected compared other oils. Storage conditions also showed the significant effect $(p<0.05)$ on the PV of the blended oils (Table 3). For IV, unblended PO, SO and SSO were significantly affected by storage conditions whereby SO showed a wider change in IV compared to other oils (Table 4). Blended oils were also significantly affected $(p<0.05)$ by storage conditions (Table 4$)$. The change of RI for the unblended PO, SO and SSO and their blends are presented in Table 5.

Table 1: Change in acid value after 60 days of storage time.

\begin{tabular}{lcc}
\hline SAMPLE & \multicolumn{1}{c}{ D } & A \\
\hline PO & $4.01 \pm 0.03^{\mathrm{a}, \mathrm{b}}$ & $4.28 \pm 0.09^{\mathrm{a}, \mathrm{b}}$ \\
SO & $0.27 \pm 0.26^{\mathrm{a}, \mathrm{b}}$ & $0.35 \pm 0.00^{\mathrm{a}, \mathrm{b}}$ \\
SSO & $0.19 \pm 0.02^{\mathrm{a}, \mathrm{b}}$ & $0.30 \pm 0.01^{\mathrm{a}, \mathrm{b}}$ \\
PO:SO (3:1) & $2.97 \pm 0.02^{\mathrm{a}, \mathrm{b}}$ & $3.03 \pm 0.006^{\mathrm{a}, \mathrm{b}}$ \\
PO:SO (1:1) & $1.58 \pm 0.04^{\mathrm{a}, \mathrm{b}}$ & $1.80 \pm 0.034^{\mathrm{a}, \mathrm{b}}$ \\
PO:SO (1:3) & $1.18 \pm 0.02^{\mathrm{a}, \mathrm{b}}$ & $1.43 \pm 0.006^{\mathrm{a}, \mathrm{b}}$ \\
PO:SSO (3:1) & $3.90 \pm 0.01^{\mathrm{a}, \mathrm{b}}$ & $4.15 \pm 0.41^{\mathrm{a}, \mathrm{b}}$ \\
PO:SSO (1:1) & $2.61 \pm 0.01^{\mathrm{a}, \mathrm{b}}$ & $2.46 \pm 0.01^{\mathrm{a}, \mathrm{b}}$ \\
PO:SSO (1:3) & $1.73 \pm 0.11^{\mathrm{a}, \mathrm{b}}$ & $1.80 \pm 0.10^{\mathrm{a}, \mathrm{b}}$ \\
PO:SO:SSO (1:1:1) & $1.39 \pm 0.006^{\mathrm{a}, \mathrm{b}}$ & $1.55 \pm 0.006^{\mathrm{a}, \mathrm{b}}$ \\
PO:SO:SSO (2:1:1) & $2.08 \pm 0.023^{\mathrm{a}, \mathrm{b}}$ & $1.32 \pm 0.052^{\mathrm{a}, \mathrm{b}}$ \\
PO:SO:SSO (1:2:1) & $1.09 \pm 0.001^{\mathrm{a}, \mathrm{b}} 0.24^{\mathrm{a}, \mathrm{b}}$ \\
PO:SO:SSO (1:1:2) & $1.03 \pm 0.003^{\mathrm{a}, \mathrm{b}}$ & $1.27 \pm 0.05^{\mathrm{a}, \mathrm{b}}$ \\
\hline Tabulated values are mean \pm SD \\
$b=$ mean values in the same column are significantly different, $p<0.05$ \\
$a=$ mean values in the same row are significantly different, $p<0.05$ \\
$\begin{array}{l}\text { S Samples stored in air tight-dark condition for } 60 \text { days } \\
\text { A }=\text { Samples stored at air free-ambient temperaturefor } 60 \text { days }\end{array}$
\end{tabular}


Table 2: Change in saponification value after 60 days of storage time.

\begin{tabular}{lcc}
\hline SAMPLE & D & A \\
\hline PO & $29.53 \pm 0.55^{\mathrm{a}}$ & $33.44 \pm 0.09$ \\
SO & $29.61 \pm 0.07^{\mathrm{a}}$ & $32.37 \pm 0.54$ \\
SSO & $17.16 \pm 0.04$ & $19.25 \pm 0.19$ \\
PO:SO (3:1) & $26.85 \pm 0.48$ & $30.75 \pm 0.02$ \\
PO:SO (1:1) & $23.25 \pm 0.05$ & $25.85 \pm 0.11$ \\
PO:SO (1:3) & $22.16 \pm 0.06$ & $23.11 \pm 0.06$ \\
PO:SSO (3:1) & $23.29 \pm 0.01$ & $24.24 \pm 0.11$ \\
PO:SSO (1:1) & $18.23 \pm 0.07$ & $21.14 \pm 0.47$ \\
PO:SSO (1:3) & $16.08 \pm 0.5$ & $17.84 \pm 0.17$ \\
PO:SO:SSO (1:1:1) & $26.86 \pm 0.04$ & $29.38 \pm 0.04$ \\
PO:SO:SSO (2:1:1) & $27.1 \pm 0.43$ & $29.79 \pm 0.01$ \\
PO:SO:SSO (1:2:1) & $19.44 \pm 0.11$ & $20.39 \pm 0.23$ \\
PO:SO:SSO (1:1:2) & $18.87 \pm 0.34$ & $19.90 \pm 0.34$ \\
\hline
\end{tabular}

Tabulated values are mean $\pm S D$

$a=$ mean values in the same column are not significantly different, $p>0.05$

$D=$ Samples stored in air tight-dark condition for 60 days

$A=$ Samples stored at air free-ambient temperature for 60 days

Table 3: Change in peroxide value after 60 days of storage time.

\begin{tabular}{lcc}
\hline SAMPLE & D & A \\
\hline PO & $2.57 \pm 0.24^{\mathrm{a}, \mathrm{b}}$ & $7.27 \pm 0.14^{\mathrm{a}, \mathrm{b}}$ \\
SO & $91.74 \pm 0.01^{\mathrm{a}, \mathrm{b}}$ & $162.08 \pm 0.11^{\mathrm{a}, \mathrm{b}}$ \\
SSO & $34.97 \pm 0.02^{\mathrm{a}, \mathrm{b}}$ & $78.51 \pm 0.09^{\mathrm{a}, \mathrm{b}}$ \\
PO:SO (3:1) & $14.24 \pm 0.01^{\mathrm{a}, \mathrm{b}}$ & $20.44 \pm 0.03^{\mathrm{a}, \mathrm{b}}$ \\
PO:SO (1:1) & $39.24 \pm 0.14^{\mathrm{a}, \mathrm{b}}$ & $50.46 \pm 0.04^{\mathrm{a}, \mathrm{b}}$ \\
PO:SO (1:3) & $57.3 \pm 0.001^{\mathrm{a}, \mathrm{b}}$ & $75.59 \pm 0.18^{\mathrm{a}, \mathrm{b}}$ \\
PO:SSO (3:1) & $5.31 \pm 0.02^{\mathrm{a}, \mathrm{b}}$ & $8.99 \pm 0.02^{\mathrm{a}, \mathrm{b}}$ \\
PO:SSO (1:1) & $11.67 \pm 0.03^{\mathrm{a}, \mathrm{b}}$ & $15.57 \pm 0.08^{\mathrm{a}, \mathrm{b}}$ \\
PO:SSO (1:3) & $13.73 \pm 0.08^{\mathrm{a}, \mathrm{b}}$ & $27.26 \pm 0.02^{\mathrm{a}, \mathrm{b}}$ \\
PO:SO:SSO (1:1:1) & $21.45 \pm 0.15^{\mathrm{a}, \mathrm{b}}$ & $54.15 \pm 0.12^{\mathrm{a}, \mathrm{b}}$ \\
PO:SO:SSO (2:1:1) & $29.71 \pm 0.03^{\mathrm{a}, \mathrm{b}}$ & $30.05 \pm 0.05^{\mathrm{a}, \mathrm{b}}$ \\
PO:SO:SSO (1:2:1) & $52.26 \pm 0.42^{\mathrm{a}, \mathrm{b}}$ & $63.42 \pm 0.22^{\mathrm{a}, \mathrm{b}}$ \\
PO:SO:SSO (1:1:2) & $26.44 \pm 0.24^{\mathrm{a}, \mathrm{b}}$ & $45.02 \pm 0.12^{\mathrm{a}, \mathrm{b}}$ \\
\hline
\end{tabular}

Tabulated values are mean $\pm S D$

$b=$ mean values in the same column are significantly different, $p<0.05$

$a=$ mean values in the same row are significantly different, $p<0.05$

$D=$ Samples stored in air tight-dark condition for 60 days

$A=$ Samples stored at air free-ambient temperature for 60 days 
Table 4: Change in iodine value after 60 days of storage time.

\begin{tabular}{lcc}
\hline SAMPLE & D & A \\
\hline PO & $16.14 \pm 0.59$ & $20.15 \pm 0.02$ \\
SO & $30.4 \pm 0.02$ & $41.39 \pm 0.03$ \\
SSO & $21.73 \pm 0.04$ & $23.06 \pm 0.06$ \\
PO:SO $(3: 1)$ & $23.12 \pm 0.01$ & $26.45 \pm 0.24$ \\
PO:SO $(1: 1)$ & $26.90 \pm 0.10$ & $27.03 \pm 0.13$ \\
PO:SO (1:3) & $27.81 \pm 0.01$ & $30.62 \pm 0.10$ \\
PO:SSO (3:1) & $19.58 \pm 0.05^{\mathrm{a}}$ & $21.47 \pm 0.05^{\mathrm{c}}$ \\
PO:SSO (1:1) & $19.73 \pm 0.01^{\mathrm{a}}$ & $21.47 \pm 0.01^{\mathrm{c}}$ \\
PO:SSO (1:3) & $20.71 \pm 0.02$ & $22.69 \pm 0.003$ \\
PO:SO:SSO $(1: 1: 1)$ & $28.18 \pm 0.05^{\mathrm{b}}$ & $30.02 \pm 0.07$ \\
PO:SO:SSO (2:1:1) & $28.40 \pm 0.29^{\mathrm{b}}$ & $31.96 \pm 0.19$ \\
PO:SO:SSO $(1: 2: 1)$ & $19.40 \pm 0.001$ & $21.54 \pm 0.01$ \\
PO:SO:SSO $(1: 1: 2)$ & $17.50 \pm 0.01$ & $19.19 \pm 0.09$ \\
\hline
\end{tabular}

Tabulated values are mean $\pm S D$

$a, b, c=$ mean values in the same column are not significant different, $p>0.05$

$D=$ Samples stored in air tight-dark condition for 60 days

$A=$ Samples stored at air free-ambient temperature for 60 days

Table 5: Change in refractive index after 60 days of storage time.

\begin{tabular}{lcc}
\hline SAMPLE & D & A \\
\hline PO & $0.0008 \pm 0.002^{\mathrm{a}, \mathrm{b}}$ & $0.0015 \pm 0.0002^{\mathrm{a}, \mathrm{b}}$ \\
SO & $0.0008 \pm 0.002^{\mathrm{a}, \mathrm{b}}$ & $0.001 \pm 0.0024^{\mathrm{a}, \mathrm{b}}$ \\
SSO & $0.0008 \pm 0.0007^{\mathrm{a}, \mathrm{b}}$ & $0.0013 \pm 0.023^{\mathrm{a}, \mathrm{b}}$ \\
PO:SO (3:1) & $0.0012 \pm 0.002^{\mathrm{a}, \mathrm{b}}$ & $0.002 \pm 0.002^{\mathrm{a}, \mathrm{b}}$ \\
PO:SO (1:1) & $0.0012 \pm 0.0005^{\mathrm{a}, \mathrm{b}}$ & $0.0014 \pm 0.00023^{\mathrm{a}, \mathrm{b}}$ \\
PO:SO (1:3) & $0.0013 \pm 0.0005^{\mathrm{a}, \mathrm{b}}$ & $0.0017 \pm 0.0005^{\mathrm{a}, \mathrm{b}}$ \\
PO:SSO (3:1) & $0.0007 \pm 0.0002^{\mathrm{a}, \mathrm{b}}$ & $0.0012 \pm 0.0024^{\mathrm{a}, \mathrm{b}}$ \\
PO:SSO (1:1) & $0.0007 \pm 0.0002^{\mathrm{a}, \mathrm{b}}$ & $0.0013 \pm 0.002^{\mathrm{a}, \mathrm{b}}$ \\
PO:SSO (1:3) & $0.0005 \pm 0.0005^{\mathrm{a}, \mathrm{b}}$ & $0.0012 \pm 0.0002^{\mathrm{a}, \mathrm{b}}$ \\
PO:SO:SSO (1:1:1) & $0.0009 \pm 0.0002^{\mathrm{a}, \mathrm{b}}$ & $0.0012 \pm 0.0002^{\mathrm{a}, \mathrm{b}}$ \\
PO:SO:SSO (2:1:1) & $0.0008 \pm 0.0004^{\mathrm{a}, \mathrm{b}}$ & $0.0013 \pm 0.00022^{\mathrm{a}, \mathrm{b}}$ \\
PO:SO:SSO (1:2:1) & $0.0012 \pm 0.00016^{\mathrm{a}, \mathrm{b}}$ & $0.0015 \pm 0.00016^{\mathrm{a}, \mathrm{b}}$ \\
PO:SO:SSO (1:1:2) & $0.0009 \pm 0.0002^{\mathrm{a}, \mathrm{b}}$ & $0.0012 \pm 0.0002^{\mathrm{a}, \mathrm{b}}$ \\
\hline
\end{tabular}

Tabulated values are mean $\pm S D$

$a=$ mean values in the same column are not significantly different, $p>0.05$

$b=$ mean values in the same row are not significantly different, $p>0.05$

$D=$ Samples stored in air tight-dark condition for 60 days

$A=$ Samples stored at air free-ambient temperature for 60 days 


\section{DISCUSSION}

\section{Acid value (AV)}

Change in AV of oils as presented in Table lindicated unblended PO, SO and SSO to have significant difference $(p<0.05)$ in an increase of FFA content after 60 days of storage time in all storage conditions whereby SSO appeared to be the most hydrolytically stable oils. The stability of SSO could be attributed by the presence of high content of natural antioxidants such as sesamin, sesamolin, sesamol and $\gamma$-tocopherol (Yan et al., 2000; Jeng and Hou, 2005; Sadeghi et al., 2009) that could be retarding formation of FFA through oxidative pathway. The increase of FFA content for the samples stored in air free-ambient temperature was significantly higher than those stored in air tight-dark conditions. The observed difference in FFA value increment for two different storage conditions could be due to the synergistic effects of both oxygen and light contacting the samples in the air free-ambient temperature storage conditions (Naghshineh et al., 2009).

The FFA change of blends in air freeambient temperature appeared to be significantly higher than those of the same blends stored in air tight-dark condition (Table 1). Binary blends of PO:SO and PO:SSO showed significant difference in an increase of FFA content in both storage conditions whereby blends of PO:SO appeared to be hydrolytically most stable than blends of PO:SSO whereby PO:SO (1:3) showed the highest stability while PO:SSO (3:1) was the least stable. For the ternary blending, PO:SO:SSO (1:1:2) blend showed the highest stability by showing the small change in FFA content followed by PO:SO:SSO (1:2:1) while PO:SO:SSO $(2: 1: 1)$ was the least stable in all storage conditions. The stability of these blends could have been attributed by the high degree of unsaturation and higher antioxidant capacity of SO and SSO which retards hydrolysis of triglycerides. Therefore, blending of saturated oils such as PO with unsaturated ones such as SO and SSO improves the stability of the former against hydrolytic degradation.

\section{Saponification value (SV)}

The storage conditions had significant effect $(p<0.05)$ on the SV of unblended PO, SO and SSO (Table 2). The increase of SV of unblended PO, SO and SSO stored at air freeambient temperature was significantly higher than the SV of the same samples stored in the dark due to synergistic effect of light and air which accelerates the hydrolysis of oils resulting into an increase of fatty acids, hence increasing SV. In both storage conditions, unblended SSO showed the highest stability than PO and SO probably due to its high content of natural antioxidants.

The increased SV observed in blends stored in air free-ambient temperature appeared to be significantly higher than the SV recorded for blends stored in the air tightdark condition (Table 2). The increased SV could be contributed by the same reasons stated earlier for unblended oils. For the binary blends, PO:SSO blends were more stable than PO:SO blends stored in the same storage conditions whereby PO:SSO (1:3) blend was the most stable followed by PO:SSO (1:1) blend. PO:SO (3:1) blend was found to be the least stable. Similarly, ternary blends with large amount of SSO were more stable than those with large amount of PO. Thus, the PO:SO:SSO (1:1:2) blend was significantly stable over the others, followed by PO:SO:SSO (1:2:1) blend containing substantial amount of highly unsaturated SO contributing to its stability against hydrolysis. The stability of the SSO containing blends are ascribed to high amount of the natural antioxidants contents which retard the formation of free fatty acids.

\section{Peroxide value (PV)}

The PV of unblended PO, SO and SSO showed an increase in both storage conditions, PO being marginally least affected compared other oils (Table 3 ). The oxidative stability of PO could be due to the low content of linoleic acid (7.5-10.9\%) as compared to estimated amounts present in SO (53-68\%) and SSO (45\%) (Ngassapa and Othman, 2001) since linoleic and linolenic acids are the most 
readily oxidized components of the oil. The samples stored at the air free-ambient temperature significantly showed a wider PV change compared to those of the sealed samples stored in the dark. This dramatic increase of PV could be attributed to the synergistic effects of oxygen and light contacting the sample thereby prompting oxidation process (Siddique et al., 2010).Thus, light and air should be avoided for proper preservation of the oils to lower their susceptibility to peroxide formation.

The PV increased in blended oils stored in the air free-ambient temperature was significantly higher than those observed in samples stored in the air tight-dark conditions (Table 3). The binary blends of PO and SSO had significantly higher stability than the corresponding blends of $\mathrm{PO}$ and $\mathrm{SO}$ stored in the same storage condition. Among the blends, PO:SSO (3:1) was the most stable followed by PO:SSO $(1: 1)$ in all storage conditions. For the ternary blends, those with high amount of SO were found to have higher PV change while those with high amount of PO had lower PV change. The increased PV of the blends at the air free-ambient temperature was significantly higher than those stored in the air-tight dark conditions (Table 3). This trend indicated the influence of light and air on peroxidation of oils, the factors that should be avoided for proper preservation of oils to lower their susceptibility to peroxide formation.

\section{Iodine value (IV)}

Changes in IV of oils and their blends after 60 days of storage under the air tight-dark conditions and at the air free-ambient temperature indicated unblended $\mathrm{PO}, \mathrm{SO}$ and SSO to be significantly affected by storage conditions whereby SO showed a wider change compared to other oils (Table 4). The IV change for the oils stored at the air freeambient temperature observed to be significantly higher than those stored in the air tight-dark conditions. It was expected that the IV would decrease with exposure to air and light due to the decrease of unsaturation.
However, the increasing trend was observed, similar to previously reported study on blends of the refined palm olein with other vegetable oils (Siddique et al., 2010). This could be contributed by the oxidation of oil constituents such as presence of some heavy metals and formation of peroxides probably enhanced by microbial activities along with air and light exposure.

The IV change observed in blends stored in air free-ambient temperature were significantly higher than the corresponding blends stored in the air tight-dark condition (Table 4). The binary blends of PO:SSO had small IV change compared to corresponding blends of PO:SO stored in the same storage conditions indicating the vulnerability of $\mathrm{SO}$ to oxidation. Both PO:SSO $(3: 1)$ and PO:SSO $(1: 1)$ showed insignificant difference $(p>0.05)$ in the corresponding storage condition being the most stable blend than others. For the ternary blends, the results showed that the blend with high ratio of PO had wider IV change than those with high ratio of $\mathrm{SO}$ and SSO in which PO:SO:SSO $(1: 1: 1)$ and PO:SO:SSO (2:1:1) blends stored in air tightdark condition showed insignificant different $(p>0.05)$.

\section{Refractive index (RI)}

The change of RI for the unblended $\mathrm{PO}$, SO and SSO stored at air free-ambient temperature was insignificantly higher $(\mathrm{p}>$ 0.05) than those in the air tight-dark conditions (Table 5). However, PO appeared to be slightly affected by light and air than SO and SSO. All blends which were stored at air free-ambient temperature showed an increase of RI which were higher than those observed in the corresponding blends stored in the air tight-dark conditions. The binary blends of $\mathrm{PO}$ and SSO showed higher stability compared to the corresponding binary blends of $\mathrm{PO}$ and $\mathrm{SO}$ kept in the same storage condition, the stability being attributed to high amount of natural antioxidants in SSO. For the ternary blends, PO:SO:SSO (1:1:2) was the most stable indicating the smallest change in RI compared to other blends. Nonetheless, the 
blended oils were not significantly affected by storage conditions $(p>0.05)$. Thus, RI could be used in identification of the initial quality of oils, but less important in determination of deterioration during storage.

\section{Conclusion}

Samples stored at the air-tight dark conditions remained relatively stable than those at the air free-ambient temperature, with sesame oil (SSO) showing the highest stability under the studied storage conditions. Generally, the unblended oils were found to have poor storage stability than the blended oils due to the synergistic effect of light and air. The study undertaken reveals that blends with higher proportions of SSO had better stability than those with high contents of PO and SO. This could have been contributed by the large amount of natural antioxidants present in SSO. PO:SO:SSO (1:1:2) blend showed the highest stability against hydrolysis indicating small change in FFA and SV than others after 60 days of storage time at both storage conditions. On the other hand PO:SSO (3:1) blend showed highest stability against oxidation by showing small change of PV in both storage conditions. Hence, such traditionally processed vegetable oils should be specially packed to keep them free from the light and air for prolonged storage. Each oil type studied had some attributes of good quality and stability; hence as anticipated the study further reveals the significance of blending in improving the quality and stability of these traditionally processed vegetable oils.

\section{COMPETING INTERESTS}

The authors declare that they have no competing interests.

\section{AUTHORS' CONTRIBUTIONS}

FNN and SSN conceived the study and designed the experiments. TRM performed the experiments. All authors participated in data analysis, discussion of results and the manuscript setup. TRM wrote the first draft of the paper; all authors improved the subsequent drafts and agreed on the final version before submission.

\section{ACKNOWLEDGEMENTS}

The Tanzanian Government through the Public Service Management in the President's Office is greatly appreciated for the financial supports granted for this research.

\section{REFERENCES}

Abdulkarim SM, Myat MW, Ghazali, HM. 2010. Sensory and physicochemical qualities of palm olein and sesame seed oil blends during frying of banana chips. J. Agric. Sci., 2(4):18-29.

Finley JW, Shahidi F. 2001. The chemistry, processing and health benefits of highly unsaturated fatty acids: An overview. American Chemical Society Symposium, Series 788, 2-11.

Jeng KCG, Hou RCW. 2005. Sesamin and Sesamolin: Nature's Therapeutic Lignans. Bentham Science Publishers Ltd: Sharjah, U.A.E.

Lee JY, Hwang DH. 2008. Dietary fatty acids and eicosanoids. In Fatty Acids in Foods and their Health Implications, ChowCK. (Ed.). CRC Press: London, UK; 713739.

Li Y, Ye M, Liu HW, Ji X H, Yan YN. 2000. Identification and determination of $(+)-$ sesamin in Semen cuscutae by capillary GC and GC-MS. Chin. Chem. Lett., 11(12): 1073-1076.

Myat MN, Abdulkarim S M, Ghazali HM, Roselina K. 2009. Physicochemical and sensory characteristics of palm olein\& peanut oil blends. J. Food Agric. Environ., 7(3\&4): 175-181.

Naghshineh M, Ariffin AA, Ghazali HM, Mirhosseini H, Kuntom A, Mohammad A. 2009. Monitoring the change of physicochemical properties of oil blend as function of storage time. $J$. food Agric. Environ., 7(3\&4): 120-125.

Ngassapa FN, Nyandoro SS, Mwaisaka TR. 2012. Effects of temperature on the physicochemicalproperties of 
traditionally processed vegetable oils and their blends. Tanz. J. Sci., 38(3): 166-176.

Ngassapa FN, Othman OC. 2001. Physicochemical characteristics of some locally manufactured edible vegetable oils marketed in Dar essalaam. Tanz. J. Sci., 27: 49-58.

Sadeghi N, Oveisi MR, Hajimahmood M, Jannat B, Mazaheri M, Mansouri S. 2009. The contents of sesamol in Iranian sesame seeds. Iran. J. Pharm. Res., 8(2): 101-105.

Sampath H, Ntambi JM. 2008. Role of stearoyl-CoA desaturase in human metabolic disease. Future Lipidol., 3(2): 163-173.

Serjouie A, Tan CP, Mirhossein H, Man YBC. 2010. Effect of vegetable-based oil blends on physical chemical properties of oils during deep-fat frying. Am. $J$. Food Techno., 5(5): 310-323

Siddique BM, Ahmad A, Ibrahim MH, Hena S. 2010. Physico-chemical properties of blends of palm olein with other vegetable oils. Grasas Aceites., 61(4): 423-429.

Sulle E, Nelson F. 2009. Biofuels, Land Access and Rural Livelihood in Tanzania. IIED, London.

TZS 51. 1979. Tanzania Standard Oils and Fats-Sampling and Test Methods Part 1: Sampling and Chemical Tests, Tanzania Bureau of Standards.

TZS 561. 2001. Tanzania Standards, Sampling and Test Methods for Palm Oil and Palm Oil Products, Tanzania Bureau of Standards.

WHO. 2003. Diet, Nutrition and the Prevention of Chronic Diseases. Geneva. 